\title{
Desenvolvimento Motor. Notas breves sobre o estado de conhecimento e propostas de pesquisa.
}

\author{
José António Ribeiro Maia \\ Faculdade de Ciências do Desporto e de Educação Física, \\ Universidade do Porto
}

https://doi.org/10.5628/rpcd.01.01.65

É óbvio que este texto não pretende percorrer o lato domínio do Desenvolvimento Motor, apresentando o seu quadro conceptual, o enfoque diverso da pesquisa actual, tão-pouco rasgar as novidades dos horizontes que norteiem a pesquisa (os interessados nestas matérias podem consultar, por exemplo, Maia, 1999a; 1999b; 2000a; 2000b; Smith e Thelen, 1993).

Ao invés, o nosso propósito é bem mais simples e limitado. Trata, tão somente, da apresentação de um sumário de alguns dos eventos da maior relevância na pesquisa no Desenvolvimento Motor em áreas como o crescimento somático, a maturação biológica, a performance motora e as habilidades motoras. É evidente que tais eventos foram marcados por homens e mulheres que lhes deram vida, o que significa que é também deles que falaremos. A grandeza, significado e alcance do estudo do crescimento somático obriga a que tal matéria extravase rapidamente o lato domínio da Biologia Humana para se dirigir a outros territórios de confluência e interesses, como são, por exemplo, a Antropologia Física, a Pediatria, a Ergonomia e as Ciências do Desporto.

O crescimento somático pode ser definido como um processo em que os indivíduos alteram de forma contínua a magnitude do seu tamanho e forma corporal num dado intervalo de tempo.

A história desta anatomia em movimento foi brilhantemente contada, interpretada e explicada pelo mais famoso auxologista do nosso tempo, o Prof. James Tanner, num livro magnífico, verdadeira epístola sem paralelo - a history of the study of human growth, bem como por Edith Boyd num volume de rara elegância, universalidade de compreensão e abordagem - origins of the study of human growth.

\author{
Vítor Pires Lopes \\ Escola Superior de Educação, \\ Instituto Politécnico de Bragança
}

Em 1749, nos volumes 2 e 3 da sua História Natural, o conde de Buffon apresenta o primeiro estudo longitudinal da história - o do filho do seu amigo conde de Montbeillard. Contudo, teríamos que esperar por Adolphe Quetelet (1796-1874) para ser formalizado, pela primeira vez, um modelo matemático para descrever este processo. Nasce nesta aventura Queteletiana, um novo ramo da Auxologia, o da modelização matemática do crescimento somático. De Quetelet a Hassane Abidi, vão cerca de 140 anos e mais de 200 formulações de tal processo, de que destacamos os modelos estruturais mais famosos e actuais - o de PreeceBaines, o Logístico Triplo e o JPA. Estes modelos permitiram mergulhar numa aventura fascinante de grandes implicações - a da previsão da estatura adulta (sobre esta matéria ver Cameron, 1995; Abidi, 1991).

Do percurso histórico do território Auxológico aparece, como estrela maior, Franz Boas que James Tanner considera, justamente, como o pai e a mãe da Auxologia. Entre os seus contributos únicos emergem de forma singular:

- O conceito de idade fisiológica ou de desenvolvimento. Daqui decorre a ideia fulcral de "tempo" de crescimento para descrever a velocidade do processo - nalgumas crianças é pianíssimo, noutras é moderatto e, noutras ainda é molto vivace.

- É o primeiro autor a perceber o significado da variação em torno dos valores centrais, aplicando e generalizando o uso da Estatística ao estudo do crescimento.

- Descreve e interpreta as curvas da velocidade, 
ainda que não as designe deste modo; identifica o pico de velocidade do crescimento e a sua variação, posteriormente retomado, expandido e interpretado no seu forte alcance por Frank Shuttleworth.

- Diferencia nitidamente curvas individuais de curvas médias baseadas em dados populacionais, estabelecendo os alicerces da construção de cartas de crescimento, principalmente as tempocondicionais.

- É o primeiro pesquisador a analisar, de forma adequada, dados longitudinais.

Uma outra estrela maior, pelo alcance da sua obra magistral, um verdadeiro livro no espírito renascentista do seu alcance e profundidade, on growth and form, é Sir D'Arcy Thompson (1994) A sua teoria das transformações, inspirada no famoso geómetra e pintor Albercht Durer, é aplicada ao crescimento. A expressão "velocidade de crescimento" cunhada por D'Arcy Thompson é introduzida por James Tanner no léxico auxológico. Da sua teoria das transformações, Julian Huxley e Georges Teissier desenvolvem, independentemente, a teoria Alométria para estudar o crescimento diferencial, que mais tarde Asmussen e HeebolNielsen (1954) aplicam, pela primeira vez às pesquisas da performance motora em seres da mesma espécie mas de tamanhos diferentes. A riqueza interpretativa da Alometria é por demais evidente nos territórios da Antropologia Física, Auxologia, Fisiologia e Ciências do Desporto (sobre esta matéria consultar Maia, 2000b).

É evidente que no vasto firmamento da Auxologia há outras estrelas maiores, como sejam Nancy Bayley, Walter Krogman, Stanley Marion Garn e Alex Roche. Contudo, a parte visível do firmamento actual é percorrida pela enorme luz que é James Mourilyan Tanner. É mais que óbvio que este não é o momento para demonstrar o apreço e admiração pelo trabalho hercúleo do Prof. Tanner. Contudo, não podemos deixar de aproveitar esta oportunidade para apresentar alguns marcos da sua carreira e impulso inesgotável no vasto campo da Auxologia. Do ponto de vista da investigação empírica, uma das suas obras mais notáveis é o famoso estudo longitudinal de Harpender, não só pela sua concepção e estrutura metodológica, mas sobretudo pela equipa que soube reunir à sua volta da qual saíram enormes inovações nos domínios do pensamento e acção Auxológicas.

A título de mero exemplo deixamos, aqui, a referência de obras maiores da literatura Auxológica da mão criativa deste homem notável:

- Growth at adolescence (1962)

- Worldwide variation in human growth (1976)

- Foetus into man (1978)

- Atlas of children growth. Normal variation and growth diseases (1982)

Em 1995, por ocasião do seu $75^{\circ}$ aniversário, colegas e alunos de 20 países produzem uma jóia singular na literatura Auxológica - essays on auxology presented to James Mourilyan Tanner (Hauspie, Lindgren e Falkner, 1995). Uma homenagem, entre muitas, a um homem a quem estaremos continuamente gratos pela luminosidade do seu percurso na Auxologia do nosso tempo, sobretudo pela sua convicção de que o estado de crescimento de uma população reflecte a justeza das suas políticas sócio-económicas.

A investigação em Portugal sobre a matéria do crescimento somático é exclusivamente e excessivamente fotográfica. Confesso que esta abordagem já nada traz de novo, tão-pouco inventaria algo que não seja óbvio. Sobre esta matéria, e se a pesquisa se debruçar exclusivamente sobre ele, é evidente a exigência de um filme com um bom elenco e um guião excelente.

Pensamos que é urgente, pelo menos, a realização de duas tarefas que do nosso ponto de vista, são da maior relevância:

$1^{\circ} \mathrm{Um}$ estudo que estabeleça normas de crescimento linear e ponderal para a população portuguesa. Já basta de ignorância sobre este assunto, exactamente ao contrário do que acontece no contexto europeu.

$2^{\circ}$ Estudos de natureza longitudinal e estudos longitudinais-mistos de implicações latas. Um exemplo esclarecedor sobre esta matéria é o que se está a realizar na Madeira, conduzido por Duarte Freitas, sob a orientação de um docente da Faculdade de Ciências do Desporto e de 
Educação Física da Universidade do Porto e do Prof. Gaston Beunen da Universidade Católica de Lovaina. Esta pesquisa exemplar no contexto português e mundial, dedica-se ao estudo interactivo de 4 grandes territórios - crescimento somático, maturação biológica, aptidão física e actividade física habitual.

Mesmo considerado no domínio biológico, a expressão maturação biológica apresenta alguma dificuldade de definição rigorosa e delimitada no seu alcance. Maturação biológica não significa espontaneidade de um resultado biológico num dado ponto do tempo. Antes reflecte a essência de um fenómeno biológico condicionado pelo factor tempo, regulado pela matriz genética do sujeito em interacção contínua e decisiva com o envolvimento. Maturação espelha, pois, duas noções centrais transformação progressiva e temporalidade intrinsecamente ligadas ao alvo da flecha que é o estado adulto do sujeito.

Um dos textos mais brilhantes realizados por James Tanner e Phyllis Eveleth em 1990 é o worldwide variation in human growth. O capítulo 8 trata da temática da maturação ao nível inter-populacional de um modo altamente exaustivo, de uma forma didáctica exemplar, evidenciando a sua forte dependência às múltiplas características do envolvimento, que Tadeuz Bielickz designou com muita propriedade de gradientes socio-económicos no crescimento e maturação. Da maior relevância é a informação acerca do declíneo da idade da menarca e suas influências psicológicas e sociais. É evidente que rapidamente extravasaremos daqui para o campo fértil da tendência secular, assunto da maior importância em termos biológicos, reflexo que é da história sócio-política e económica de um povo. Num domínio distinto de preocupações encontra-se a enorme produção de Robert Malina e Gaston Beunen. Do primeiro realçamos, sobretudo, o seu texto brilhante, um verdadeiro manual de enorme riqueza informativa - growth, maturation and physical activity (Malina e Bouchard, 1991).

Do segundo destacamos o fascinante estudo acerca do carácter interactivo do crescimento somático, estilo de vida e saúde realizado em Lovaina (Beunen et al., 1988). Trata-se de uma pesquisa praticamente sem par no domínio Auxológico aplicado à Educação Física e ao Desporto.

Um outro vasto território fortemente lavrado conjuntamente por estes autores é o que trata das seguintes parcelas:

- A primeira lida com a necessidade imperiosa de se considerar os níveis distintos de maturação para interpretar adequadamente os dados do crescimento somático e o seu desempenho motor de crianças e jovens.

- A segunda refere-se à exigência da identificação adequada do perfil multivariado do jovem atleta de sucesso, do redimensionamento dos escalões competitivos em função da idade cronológica, da resposta distinta ao treino e competição de crianças e jovens, bem como à necessidade de interpretação criteriosa da sua enorme variabilidade.

- A terceira compreende uma parcela especial, dado apresentar um carácter fortemente secante com vários outros domínios. Estamos a falar da temática altamente controversa dos períodos sensíveis e prontidão desportivo-motora não só no que se refere ao domínio substantivo como também ao metodológico Neste vasto e complexo domínio de investigação importa esclarecer o grau de sensibilidade ao treino nos diferentes níveis etários, de forma a identificar aqueles períodos onde os efeitos do treino sejam majorados. Para isso é indispensável recorrer a estudos longitudinais, e de preferência que em simultâneo sejam experimentais. Estudos com este delineamento não são conhecidos. Digase que levar um estudo deste tipo por diante é uma tarefa hercúlea que poucos se atreverão a realizar. Os únicos estudos que abordaram esta problemática foram os de Sprynarová (1974), Kobayashi et al. (1978), Weber, Kartodihardjo e Klissouras (1976) e Koch (1980) que pesquisaram o desenvolvimento da aptidão de resistência (expressa pelo $\mathrm{VO}_{2}$ ) e que chegaram a resultados contraditórios (sobre esta matéria ver em língua portuguesa Lopes e Maia, 2000).

- A quarta é a da interpretação do desempenho motor a partir do alinhamento dos dados pelo pico de velocidade da altura (pelo menos com base no modelo de Preece-Baines, se é que só existe um 
pico de velocidade de crescimento). Uma outra avenida da maior riqueza em termos descritivos e esclarecimento do seu significado é a que se refere ao uso desse instrumento de pensamento e metodologia que é a Alometria (sobre esta matéria ver Asmussen e Heebol-Nielsen, 1954; Maia, 2000b; Prista, Marques e Maia, 1999)

O domínio da performance motora tem percorrido várias avenidas de pensamento metodológico e inquietação epistemológica nos pesquisadores de diferentes interesses nas Ciências do Desporto das áreas designadas de mais "hard" como sejam, por exemplo, a biomecânica e a fisiologia, até às mais "soft" como são, a título de exemplo, a psicologia e a sociologia.

Este fascínio inesgotável pelo fenómeno da performance, enquanto processo e produto, tem produzido páginas do maior esclarecimento metodológico e de modelização sobretudo na Psicologia Industrial. Exemplos fascinantes deste empreendimento são o monumental trabalho de Fleishman e Quaintance (1984), e os textos dos congressos sobre a temática editados por Frank Landy e colaboradores (1983) e por Ronald Berk (1987). Do modelo simples de Weineck, aos modelos de enorme flexibilidade da Metodologia de Estruturas de Covariância vai um passo enorme que se impõe como provocador de interesses mais aprofundados dos pesquisadores das Ciências do Desporto (sobre esta matéria ver Maia, 1997).

$\mathrm{Na}$ área cientifica e pluridisciplinar que é o

Desenvolvimento Motor, a modelização da performance é uma tarefa ainda por realizar, quando pensamos no domínio estrito das aptidões dos sujeitos.

É fácil descrever a performance de um conjunto de sujeitos em função da sua idade cronológica. Basta para tanto servirmo-nos de modelos simples, lineares ou não. Bem mais complicado é descrever e interpretar a velocidade com que se regista a performance em diferentes pontos do tempo, i.e. estudar o comportamento da sua estabilidade e mudança.

Neste domínio temos disponíveis duas abordagens.

A primeira e única na literatura, inspirada em modelos estruturais do crescimento somático, foi proposta por Beunen, Malina e colaboradores
(1988), numa monografia a todos os títulos notável - adolescent growth and motor performance. Pela primeira vez modelam-se curvas da distância e velocidade para as Aptidões motoras. Um outro feito notável neste texto é o alinhamento dos valores da performance pelo pico da velocidade da altura e peso, na esteira das sugestões de Franz Boas, Frank Shuttleworth, James Tanner e Donald Bailey. Da sua possibilidade de representação gráfica somos tentados, sobretudo os menos experientes, em ver aí algo de substancial que nos liga ao vasto e polémico assunto dos períodos sensíveis.

A segunda abordagem, designada de análise de trajectórias latentes, é bem mais rica e flexível do que a anterior, permitindo acomodar preditores da mudança e estabilidade que sejam, ou não, invariantes no tempo. Fortemente utilizada em contextos educacionais e em psicologia do desenvolvimento, é praticamente desconhecida pelos pesquisadores portugueses. Esta proposta tem revelado enormes potencialidades no território das aptidões motoras e foi apresentada pela primeira vez no domínio das Ciências do Desporto por Maia e colaboradores (1999a; 1999b; 2000a) em Portugal, no Brasil e em contextos internacionais.

Se pensarmos em desenvolvimento de aptidões somos forçosamente obrigados a pesquisas longitudinais. Se temos dados longitudinais, o que é praticamente inexistente em Portugal, há que pensar em noções como mudança, estabilidade, velocidade, aceleração. Ora os modelos propostos não só iluminam este território algo esquecido, mas permitem, sobretudo o das trajectórias latentes, uma busca apetecível ao tesouro da mudança e do que a causa.

Um outro olhar para o mesmo território faz emergir um quadro distinto de problemas se centrarmos a nossa atenção no domínio estrito da modelação. Uma lista brevíssima desta agenda poderia orientarnos por dois planos aparentemente distintos - o das aptidões motoras e o da performance desportiva. No plano das aptidões motoras, por exemplo, é possível discernir programas fascinantes de pesquisa que poderiam ajudar a esclarecer as seguintes questões:

Será possível identificar uma estrutura da performance motora baseada nas aptidões dos 
sujeitos, no seu nível maturacional e dimensões lineares? E será tal proposta passível de testagem empírica?

Será que esta estrutura é invariante nos dois sexos e diferentes intervalos de idade?

Qual será o contributo do pensamento Alométrico para desbravar o terreno da performance condicionada pelo factor dimensional? Quais os procedimentos e estratégias metodológicas para pesquisar o fascinante problema da estabilidade e mudança dos níveis de aptidão e perfomance condicionados pela flecha do tempo?

No plano da performance desportiva, as preocupações dos investigadores teriam, forçosamente, que ajudar a esclarecer a fenómeno percorrendo, pelo menos o seguinte trajecto: inventariar e hierarquizar as qualidades/ características que discriminam atletas de nível de rendimento distinto. Situamo-nos, aqui, no domínio estrito do sujeito. É ele o objecto de análise. As variáveis a serem utilizadas deveriam ser alvo de uma crítica severa e passar pelo crivo da bibliografia mais importante, do pesquisador, do treinador e dos atletas. O recurso à análise da função discriminante, análise factorial e regressão logística podem ser do maior interesse.

A performance motora é um fenótipo extremamente complexo. A sua maior característica é expressa pela imensa variação do seu resultado no seio da população.

Dois factores são responsáveis por tal variabilidade o genótipo e o envolvimento.

Uma área de estudo de confluência da Genética com a Biometria, é a Genética Quantitativa, que ao inspirarse na metodologia Top-Down, procura interpretar tal variação, servindo-se de modelos complexos e altamente flexíveis para acomodar delineamentos distintos de que destacamos os das famílias nucleares e os gemelares (sobre esta matéria consultar Bouchard, Malina e Pérusse, 1997; Maia et al., 2000c). Ainda que não tenhamos espaço para traçar a história deste percurso nas Ciências do Desporto, há que mencionar alguns eventos principais, bem como os seus grandes mentores:
1. O fascínio pelo "homo olimpicus", fenótipo único na enorme variabilidade morfológica e "performator" do "homo sapiens sapiens", originou as primeiras pesquisas de Grebbe, Grimm, Jokl e Jokl e Gedda. Os propósitos eram não só identificar e descrever a fenótipo "homo olimpicus", mas ao mesmo tempo, verificar a existência de linhagens familiares de desportistas de sucesso, um pouco na esteira da pesquisa pioneira de Galton sobre a genialidade herdada no seio de determinadas famílias.

2. Vassilis Klissouras apresenta, pela primeira vez, na prestigiante revista Journal of Applied Physiology, em 1971, um texto, verdadeiro marco histórico, acerca da variabilidade no consumo máximo de $\mathrm{O}_{2}$ que é devida a diferenças genéticas entre sujeitos. Desde esta data que esta matéria tem sido objecto de forte debate, comandado sobretudo pela equipa de Laval, no Canadá. A pesquisa mais vasta e actual sobre este assunto é o Heritage Family Study que congrega 5 instituições do maior prestígio nos Estados Unidos e Canadá liderada pelo Prof. Claude Bouchard.

3. Conferências e publicações acerca da matéria florescem a partir dos anos 80 com o livro de Rudolf Kovar na Checoslováquia, as actas dos congressos editadas por Napoleon Wolanski na Polónia e Malina e Bouchard nos Estados Unidos.

4. Em 1992 e 1997 realizaram-se duas dissertações de doutoramento na Universidade Católica de Lovaina, sob a orientação do Prof. Gaston Beunen que são um marco fundamental desta matéria. A primeira da autoria de Hermine Maes procura pesquisar a importância dos factores responsáveis pela variação fenotípica no crescimento e aptidão física de gémeos e seus progenitores. A segunda da autoria de Martine Thomis pretende identificar a distinta sensibilidade da resposta ao treino da força em gémeos.

5. Contudo, o prestígio desta área de pesquisa é devedora, na sua projecção, a uma estrela maior no firmamento da investigação em Ciências do Desporto - Claude Bouchard. Desde as suas primeiras inquietações publicadas no famoso manual la preparation d'un champion, passando pela sua dissertação de doutoramento em Epidemiologia Genética, que juntamente com o 
seu orientador, amigo e cúmplice, o Prof. Robert Malina, têm saído as páginas mais esclarecidas, didácticas e promissoras de longo alcance no entendimento da variação da performance motora.

Exemplos esclarecedores do que acabamos de referir são, a título meramente ilustrativo, as seguintes publicações:

Genetics for the sport scientist: selected methodological considerations (1983),

Sport and human genetics (1986),

que culminaram com o texto brilhante, genetics of fitness and physical performance, resumo actual do conhecimento sobre a matéria, uma síntese única para os pesquisadores da performance e das suas causas.

Apesar do brilhantismo desta aventura inquisitiva, os investigadores portugueses das Ciências do Desporto parecem ignorar tais pesquisas e inquietações. Haverá algum motivo para esta eventual cegueira, ou será pura e simplesmente ausência de interesse?

- Será desconhecimento da matéria?

- Insuficiência de formação?

- Temor do assunto?

- Convicção que a matéria é para outros?

- Ausência de projectos de pesquisa de larga escala que compreendam famílias inteiras, bem como gémeos?

- Ou será por outros motivos plausíveis e justificados?

Para colmatar esta insuficiência está a realizar-se na Faculdade de Ciências do Desporto e de Educação Física da Universidade do Porto, uma pesquisa de larga escala (cerca de 6000 famílias nucleares) sobre a agregação familiar e os determinantes dos níveis de actividade física de jovens dos dois sexos do $5^{\circ}$ ao $12^{\circ}$ ano de escolaridade. Desta pesquisa, uma parte compreende gémeos e as suas famílias (cerca de 500 pares).

Inspirados no passado e presente da investigação em Genética Quantitativa, tomando como companheiros de viagem os Professores Sieuve Monteiro (ICBAS-
UP), Gaston Beunen, Martine Thomis, Robert Vlietinck e Ruth Loos (KUL), Michael Neale e Hermine Thomis (VCU) iremos estudar dois grandes tipos de problemas em epidemiologia genética, e que são:

O que trata do fenómeno da agregação familiar da prática do desporto e níveis diferenciados de actividade física, bem como o que naturalmente se lhe segue, e que é o da determinação das suas fontes de variação relativas à (1) susceptibilidade biológica herdada; (2) forte exposição a um envolvimento comum; (3) e herança cultural.

Um campo de investigação de grande relevância é o que se refere ao processo de aquisição e desenvolvimento das habilidades motoras e do controlo motor ou da coordenação.

As preocupações dos pioneiros desta área de conhecimento (Shirley, Ames, Halverson) centravam-se no processo de aquisição de habilidades motoras elementares (e.g. preensão intencional, marcha autónoma) e na descrição das suas fases de desenvolvimento.

O estudo do desenvolvimento das habilidades motoras foi, até à publicação do trabalho de Kugler, Kelso e Turvey (1982), marcadamente descritivo, tendo como paradigma a teoria dos estádios. Esta corrente de estudos, da qual se destacam autores como Wikstrom, Roberton, Halverson, Seefeldt e Gallahue entre outros, levou à construção de sequências de desenvolvimento de habilidades $(v . g$. Gallahue, 1982; Wiscstrom, 1983). As sequências de desenvolvimento foram elaboradas a partir de análises cinematográficas, considerando as características das relações espaço-temporais nos segmentos corporais, e referem-se a habilidades fundamentais (correr, saltar, lançar, pontapear, agarrar, rolar, etc.). A partir destas sequências foram elaboradas escalas de avaliação das habilidades básicas (e. g. McClenaghan e Gallahue, 1978). O problema do ponto de vista da investigação aplicada é que poucas sequências de desenvolvimento de habilidades motoras, com interesse para a EF e o desporto, foram validadas. A validação das sequências de desenvolvimento das habilidades requer estudos longitudinais com grandes amostras 
(Roberton, 1989), bem como o desenvolvimento de procedimentos estatísticos para estudar estes processos aparentemente discretos. Esta corrente de investigação não tem sido capaz de explicar as mudanças que ocorrem ao longo da idade no processo de aquisição e desenvolvimento das habilidades motoras.

O trabalho de Kugler, Kelso e Turvey (1982) marcou de facto uma viragem radical pela introdução da teoria dos sistemas dinâmicos no estudo do desenvolvimento das habilidades. Com este novo paradigma pretende-se conhecer quais as variáveis quer internas quer externas que influenciam o processo de aquisição e desenvolvimento das habilidades. A teoria dos sistemas dinâmicos sugere que o estabelecimento da coordenação numa habilidade consiste na emergência e integração das estruturas coordenativas como dinâmica da modificação do sistema, ocasionada pela modificação da massa, do comprimento e da transferência de energia. Assim, é possível identificar alguns aspectos a que investigação nesta área poderá dedicar especial atenção:
- A identificação e tracking das estruturas coordenativas (Roberton e Halverson, 1988). - A identificação das variáveis de controlo e de coordenação do movimento (Caldwell e Clark, 1990).

No âmbito da teoria dos sistemas dinâmicos destacam-se os estudos de Thelen (e. g. Thelen e Fisher, 1982; Thelen et al. 1982; Thelen, RidleyJhonson e Fisher, 1983; Thelen, Fisher e RidleyJhonson, 1984; Thelen, 1986) e de Clark, Philips e Petersen, 1989) que demonstram a eficácia do uso desta perspectiva para o entendimento da organização e regulação do movimento e podem apontar o caminho para a compreensão da aquisição das habilidades motoras.

Pedimos desculpa pelo carácter demasiado tangencial deste texto. A vastidão da temática tinha este risco e quisemos assumi-lo. Esperamos ter conseguido inventariar os marcos mais relevantes e as personalidades mais carismáticas. Para o espaço disponível confessamos que não fomos capazes de fazer melhor.

\section{BIBLIOGRAFIA}

Abidi, H. Contribution a la methodologie de la modelisation des courbes de croissance. Example de la croissance staturale chez l'être humain. Tese de doutoramento. Universidade Claude Bernard-Lyon I. 1991.

Ames, L. The sequential patterning of prone progression in the human infant. Genetic Psychology Monographs. 19: 409460. 1937.

Asmussen, E., Heebol-Nielsen, K.R. A dimensional analysis of physical performance and growth in boys. Journal of Applied Physiology. Vol. 7: 593-603. 1954.

Berk, R. Performance assessment: methods and applications. Johns Hopkins University Press. Baltimore. 1987.

Beunen, G.P., Malina, R.M., Van't Hof, M.A., Simons, J., Ostyn, M., Renson, R., Van Gerven, D. Adolescent growth and motor performance. A longitudinal study of belgian boys. Human Kinetics Books. Champaign. 1988.

Bouchard, C.; Brunelle, J.; Godbout, P. La preparation d'un champion. Un essai sur la preparation à la performance sportive. Editions du Pelican. Quebéc. 1973.

Bouchard, C., Malina, R. Genetics for the sport scientist: selected methodological considerations. In Terjung, R.L. (ed), Exercise and Sport Sciences Review. The Franklin Institute
Press. New York. 1983.

Bouchard, C.; Malina, R.; Pérusse, L. Genetics of fitness and physical performance. Human Kinetics Publishers.

Champaign. 1997.

Caldwell, G. E.; Clark, J. E. The measurement and evaluation of skill within the dynamical systems perspective. In: J. E. Clark e J. H. Humpherey (eds.). Advances in Motor

Development Research. Vol. 3. Ams Press. New York. 1990. Cameron, N. The prediction of adult height. In Hauspie, Lindgren e Falkner (eds), Essays on auxology presented to James Mourilyan Tanner by former colleagues and fellows. Castlemead Publications. Welwyn Garden City. 1995, pp. 126-140.

Clark, J. E.; Philips, S. J.; Petersen, R. Developmental stability in jumping. Developmental Psychology. 25 (6): 929-935. 1989 D'Arcy Thompson, W. On growth and form. An abridge edition edited by John Tyler Bonner. Cambridge University Press. Cambridge. 1994.

Eveleth, P.B., Tanner, J.M. Worldwide variation in human growth. Cambdrige University Press. Cambridge. 1976. Fleishman, E.; Quaintance, M.K. Taxonomies of human performance. The description of human tasks. Academic Press. 
New York. 1984.

Gallahue, D. L. Understanding motor developing in children. John Wiley \& Sons. New York. 1982

Halverson, H. M. An experimental study of prehension on infants by means of systematic cinema records. Gen. Pstch. Monography. v. 10: 110-186. 1931.

Halverson, H. M. The development of prehension in infants. Child Behaviour and development. MacGraw Hill. New York. 1943

Hauspie, R., Lindgren, G., Faulkner, F. (eds). Essays on auxology presented to James Mourilyan Tanner by former colleagues and fellows. Castlemead Publications. Welwyn Garden City. 1995.

Klissouras, V. Heritability of adaptative variation. Journal of Applied Physiology. 31: 338-344. 1971.

Kobayashi, K.; Kitamura, K.; Miura, M.; Sodeyama, H.; Murase, Y.; Miyashita, M.; Matsui, H. Aerobic power as related to body growth and training in japanese boys: a longitudinal study. Journal of Applied Physiology Respiratory Environment Exercise Physiology. 44 (5): 666-672. 1978.

Koch, G. Aerobic power, lung dimensions, ventilatory capacity, and muscle blood flow in 12-16-year-old boys with high physical activity. In: K. Berg; B. O. Erikson; R. C. Nelson; C. A Morehouse (eds.) Children and Exercise IX. University Park Press. Baltimore. 1980.

Kovar, R. Human variation in motor abilities and its genetic analysis. Charles University. Prague. 1981.

Kugler, P.; Kelso, J. A. S.; Turvey, M. On the control and coordination of naturally developing systems. In: J. A. S. Kelso e J. E. Clark (eds.). The development of movement control and coordination. Wiley. New York. 1982.

Landy, F.; Zedeck, S.; Cleveland, J. (eds). Performance measurement and theory. Lawrence Erlbaum Associates. Hillsdale. 1983.

Lopes, V. P. Maia, J.A.R. Períodos Críticos ou Sensíveis. Revisitar um tema polémico à luz da investigação empírica. Revista Paulista de Educação Física. (aceite para publicação). 2000. Maes, H.H.M. Univariate and multivariate genetic analysis of physical characteristics of twins and parents. Dissertação de doutoramento. Universidade Católica de Lovaina. Lovaina. 1992.

Maia, J.A.R. A modelação da performance desportivo-motora. Um contributo centrado no pensamento de Fleishman e Quaintance e na modelação da estrutura de covariância. Revista Movimento. 6:34-50. 1997

Maia, J.A.R., Beunen, G., Lefevre, J., Claessens, A. Tradução empírica do desenvolvimento das aptidões psicomotoras: um estudo comparativo entre modelos simplex e de trajectórias latentes. In Avaliação Psicológica: Formas e Contextos (Vol. VI). Braga: APPORT, pp:799-892. 1999a.

Maia, J.A.R., Beunen, G., Lefevre, J., Claessens, A. Modeling performance. Simplex (Markov and Wiener) and latent growth curve approaches. Comunicação apresentada no American College of Sports Medicine, Seattle. 1999b.

Maia, J.A.R. A modelação da ideia de desenvolvimento a partir da análise das trajectórias latentes. Um estudo aplicado às aptidões motoras. Movimento. 2000a (no prelo).

Maia, J.A.R. Relatório pedagógico da disciplina de Desenvolvimento Motor. Documento não publicado. FDEF-UP. Porto. 2000b.

Maia, J.A.R.; Loos, R.; Beunen, G.; Thomis, M.; Vlietinck, R.; Morais, F.P.; Lopes, V.P. Aspectos genéticos da prática desportiva. Um estudo em gémeos. Revista Paulista de Educação Física. No prelo. 2000c.

Malina, R., Bouchard, C. (eds). Sport and human genetics. Human Kinetics Publishers, Inc. Champaign. 1986.

Malina, R.M., Bouchard, C. Growth, maturation, and physical activity. Human Kinetics Publishers, Inc. Champaign. 1991. McClenaghan, B.; Gallahue, D. Fundamental movement: observation and assessment. W. B. Saunders. Filadélfia. 1978. Prista, A.; Marques, A.T.; Maia, J.A.R. Allometric Scaling of physical performance in children and youth from Maputo, Mozambique. Medicine and Science in Sports and Exercise. v.31, nº5:S319. 1999.

Roberton, M. A. Developmental sequence and task analysis. In J. S. Skiner; C. B. Corbin; D. M. Landers; P. E. Martin; C. L. Wells. Future directions in exercise and sport science research. Human kinetics. Champaign. 1989.

Roberton, M. A.; Halverson, L. E. The development of locomotor coordination: longitudinal change and invariance. Journal of Motor Behaviour. 20(3): 197- 241. 1988

Shirley, M. The first two years, a study of twenty-five babies. The University of Minnesota Press. Minneapolis. 1931 Smith, L.B., Thelen, E. (eds). A dynamic systems approach to development applications. The MIT Press. Cambridge. 1993. Sprynarová, S. Longitudinal study of the influence of different physical activity programas on functional capacity of the boys from 11 to 18 years. In: J. Borms; M. Hebblink (eds.). Children and Exercise V. Acta Paediatrica Belgica. 28 (suplemento). 1974 Tanner, J. M. Growth at adolescence, $2^{\text {nd }}$ edition. Blackwell Scientific. Oxford. 1962

Tanner, J.M. A history of the study of human growth Cambridge University Press. Cambridge. 1981.

Tanner, J.M. Foetus into man: physical growth from conception to maturity. Open Books. London. 1978.

Tanner, J.M., Whitehouse, R.H. Atlas of children's growth: normal variation and growth disorders. Academic Press. London. 1982.

Thelen, E. Treadmill-elicited stepping in seven-month-old infants. Child Development. 57: 1498-1506. 1986

Thelen, E.; Fisher, D. M.; Ridley-Jhonson, R. The relationship between physical growth and a newborn reflex. Infant Behavior and Development. 7: 479-493. 1984

Thelen, E.; Fisher, D. M.; Ridley-Jhonson, R.; Griffin, N. The effects of body build and arousal on newborn infant stepping. Developemental Psychology. 158: 447-453. 1982

Thelen, E.; Fisher, D. Newborn stepping: an explanation for a “disappearing reflex". Developmental Psychology. 18: 760775. 1982

Thelen, E.; Ridley-Johnson; Fisher, D. Shifting patterns of bilateral coordination and lateral dominance in the les movements of Yong Infants. Developmental Psychobiology. 16: 29-46. 1983

Thomis, M. The power of individual genetic factor scores in predicting the sensitivity to environmental stress.

Dissertação de doutoramento. Universidade Católica de Lovaina. Lovaina. 1997.

Weber, G.; Kartodihardjo, W.; Klissouras, V. Growth and physical training with reference to heredity. Journal of Applied Physiology. 40: 211-215. 1976.

Wickstrom. R. L Fundamental motor patterns. $3^{\text {a }}$ ed. Lea \& Febiger. Filadelfia. 1983

Wolanski, N. Siniarska, A. (eds). Genetics and psychomotor traits in man. Polish Academy of Sciences. Warsow. 1984. 